\title{
EM Simulation Accuracy Enhancement for Broadband Modeling of On-Wafer Passive Components
}

\author{
Johansen, Tom Keinicke; Jiang, Chenhui; Hadziabdic, Dzenan; Krozer, Viktor
}

Published in:

Proceedings of the 37th European Microwave Conference

Link to article, DOI:

10.1109/EUMC.2007.4405426

Publication date:

2007

Document Version

Publisher's PDF, also known as Version of record

Link back to DTU Orbit

Citation (APA):

Johansen, T. K., Jiang, C., Hadziabdic, D., \& Krozer, V. (2007). EM Simulation Accuracy Enhancement for Broadband Modeling of On-Wafer Passive Components. In Proceedings of the 37th European Microwave Conference: EuMIC 2007 (pp. 1245-1248). IEEE. https://doi.org/10.1109/EUMC.2007.4405426

\section{General rights}

Copyright and moral rights for the publications made accessible in the public portal are retained by the authors and/or other copyright owners and it is a condition of accessing publications that users recognise and abide by the legal requirements associated with these rights.

- Users may download and print one copy of any publication from the public portal for the purpose of private study or research.

- You may not further distribute the material or use it for any profit-making activity or commercial gain

- You may freely distribute the URL identifying the publication in the public portal 


\title{
EM Simulation Accuracy Enhancement for Broadband Modeling of On-Wafer Passive Components
}

\author{
T. K. Johansen, C. Jiang, D. Hadziabdic, V. Krozer \\ Technical University of Denmark, Oersted-DTU \\ Section of Electro-Science, 2800 Kgs. Lyngby, Denmark \\ tkj@oersted.dtu.dk
}

\begin{abstract}
This paper describes methods for accuracy enhancement in broadband modeling of on-wafer passive components using electromagnetic (EM) simulation. It is shown that standard excitation schemes for integrated component simulation leads to poor correlation with on-wafer measurements beyond the lower $\mathrm{GHz}$ frequency range. We show that this is due to parasitic effects and higher-order modes caused by the excitation schemes. We propose a simple equivalent circuit for the parasitic effects in the well-known ground ring excitation scheme. An extended L-2L calibration method is shown to improve significantly the accuracy of the on-wafer component modeling, when applied to parasitic effect removal associated with the excitation schemes.
\end{abstract}

\section{INTRODUCTION}

Integrated passive components are employed in many microwave systems. Their performance directly impacts system operation and needs therefore accurate modeling. For accurate broadband modeling of integrated passive structures using EM simulation the simulation setup must describe the real onwafer conditions as close as possible. Particular emphasis has to be put on the correct excitation of the structure being simulated. For this reason the EM simulation setup for on-wafer passive component modeling often includes a surrounding ground ring [1]-[2]. In this excitation scheme a gap source port excites an uniform electric field across a small gap between the signal conductor and the ground conductors. This excitation scheme resembles very well the measurement situation encountered with ground-signal-ground (GSG) probes in the lower $\mathrm{GHz}$ frequency range [3].

For millimeter-wave on-wafer passive component modeling, however, the parasitics caused by the ground ring and the gap source port discontinuity may be comparable to the circuit parameters of the on-wafer components themselves. To enhance the accuracy of broadband component models these effects must be carefully evaluated and removed. It should be stressed that the form of the parasitics are such that a deembedding approach using dummy open and short structures will not provide the necessary accuracy.

Previously, a lot of effort has been put on numerical deembedding of the discontinuities associated with gap source ports [4]-[5]. The port discontinuities arise due to fringing fields and transverse current flow in the vicinity of the gap source, translating into parasitic capacitances and inductances

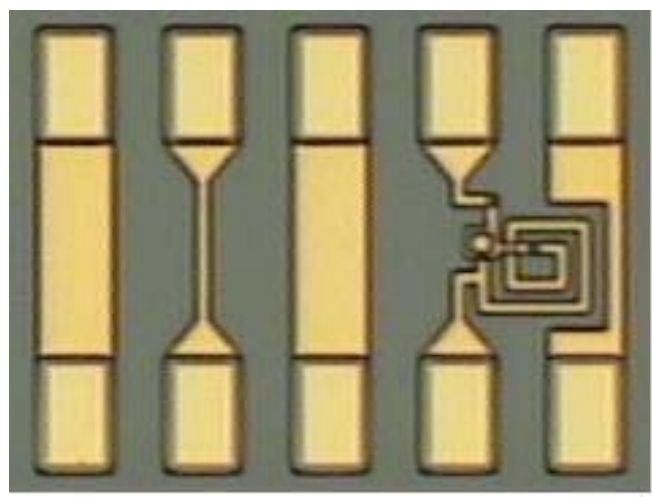

Fig. 1. Microphotograph of the on-wafer verification structures. Left: interconnect line. Right: lumped $L C$-resonator.

in a circuit model [6]. To remove the effect of port discontinuities the double-delay (L-2L) method [4] and the short-open calibration (SOC) method [5] have been proposed. An extension of these numerical de-embedding methods to on-wafer component modeling has not been previously demonstrated.

In this paper we investigate first the accuracy of three standard excitation schemes for modeling of on-wafer passive components using the commercial 3D FEM based EM simulator, Ansoft HFSS. Next we describe how an extended L-2L calibration method can be applied in connection with the 3D FEM based EM simulation to enhance the accuracy for broadband modeling of on-wafer passive components.

\section{ON-WAFER VERIFICATION STRUCTURES}

The on-wafer passive components considered for verification are depicted in Fig. 1. The verification structure shown in the left part is an interconnect line while the right part shows a lumped LC-resonator designed for a resonance frequency around $30 \mathrm{GHz}$. The lumped LC-resonator consists of a sub$\mathrm{nH}$ spiral inductor and a MIM capacitor. The structures are embedded in a finite ground coplanar waveguide (FG-CPW) transmission media with backside metallization as typically encountered in on-wafer measurements.

The verification structures are built on a $650 \mu \mathrm{m}$ thick InP 
substrate with polyimide passivation. On-wafer measurements were performed in the frequency range from $250 \mathrm{MHz}-65$ $\mathrm{GHz}$ using GSG probes exhibiting a pitch of 150um. The reference plane for the measurements was defined at the probe tips by standard SOLT ISS calibration.

\section{EM Simulation OF VERIFICATION STRUCTURES}

In this section three standard excitation schemes for simulating the on-wafer verification structures with Ansoft HFSS are discussed [3]. The excitation schemes considered are a wave-port, surrounding ground ring, and metallic box wall setup. The accuracy of these excitation schemes have mainly been verified for integrated passive components at lower microwave frequencies, e.g [1]. However, as will be shown here, once higher microwave and millimeter-wave frequencies are considered the correlation between the EM simulation results and on-wafer measurements becomes rather poor.

\section{A. EM Simulation Setups}

1) Wave-Port Solution: In the case of a wave-port excitation scheme no port discontinuity exists in the sense of parasitic capacitances and inductances. The wave-port excitation scheme should excite a single quasi-TEM CPW mode consistent with general ideas of circuit theory. It is well-known, however, that the conductor backed FG-CPW structure supports several propagating modes [7]. To ensure the excitation of the correct mode the ground conductors and backside metallization needs to be effectively tied together. For the simulation of the conductor backed FG-CPW structure this is normally assured by intersecting both the backside metallization and the ground conductors with the edge of the wave-port which processes the PEC boundary condition. The lateral size of the wave-port, however, should be larger than three times the overall CPW ground-to-ground spacing in order to terminate the electric field on the ground conductors and not on the wave-port edge. This requirement can not easily be met for the FG-CPW structures investigated in this work unless so-called wave-port fingers are employed to assure that the ground conductors are tied together. To be consistent with the reference plane during on-wafer measurements the simulated S-parameters are deembedded a distance of $40 \mu \mathrm{m}$ inwards.

2) Surrounding Ground Ring: An alternative excitation scheme for the simulation of FG-CPW's is the well-known surrounding ground ring approach [2]. A variation of this excitation scheme is shown in Fig. 2. A vertical PEC bridge connects the two ground conductors together and a lumped port is used to excite the structure. The idea is that this setup comes close to the actual on-wafer measurement condition where the GSG probe tips excite the structure from the top. Again, to be consistent with the GSG probe tip placements in the on-wafer measurements the PEC bridge is located $40 \mu \mathrm{m}$ from the edge of the structure.

3) Metallic Box Wall: Yet another method of exciting FGCPW's is to surround the structure with a metallic box as typically associated with a shielded EM simulation. A lumped port located between the center conductor and the metallic box

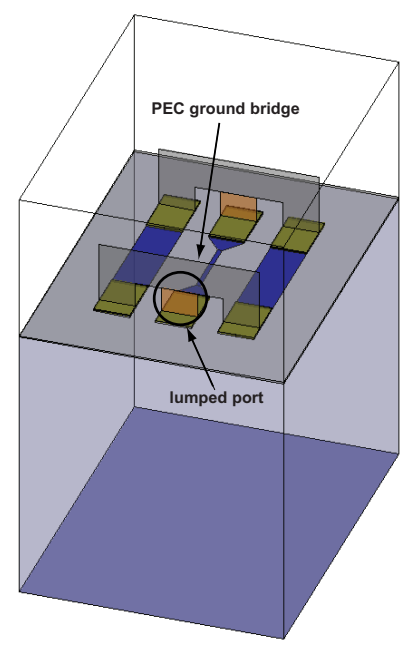

Fig. 2. HFSS setup for the surrounding ground-ring excitation. Each port lies across a gap between the center conductor and the PEC ground bridge.

wall excites the structure. The ground conductors are extended to touch the metallic box wall. The size of the metallic box should be chosen such that no box resonances are possible in the frequency range of interest. Notice that the reference plane is set at the edge of the structure in this simulation.

\section{B. EM Simulation Results}

In Fig. 3 the characteristic impedance for the interconnect line, extracted from measured S-parameters using the method described in [8], is compared with the results obtained from the respective EM simulation setups. Only the interconnect line is considered here as the sensitivity to errors originating from the excitation schemes is larger as compared to that of the lumped $L C$-resonator. Though no port discontinuity should be associated with the wave-port excitation the measurement and simulation results do not correlate well. A possible explanation of the observed behavior is as follows. In the wave-port setup the edge of the wave-port acts similar to vias in connecting

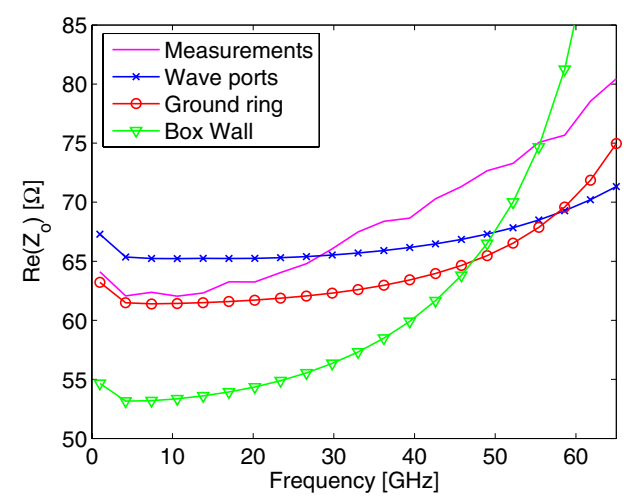

Fig. 3. Measured and simulated characteristic impedance for on-wafer interconnect line $(1 \mathrm{GHz}-65 \mathrm{GHz})$. Measurements (-), wave-port setup $(-\mathrm{o}-)$, ground ring setup (-x-), metallic box wall setup (- $\left.\nabla^{-}\right)$ 


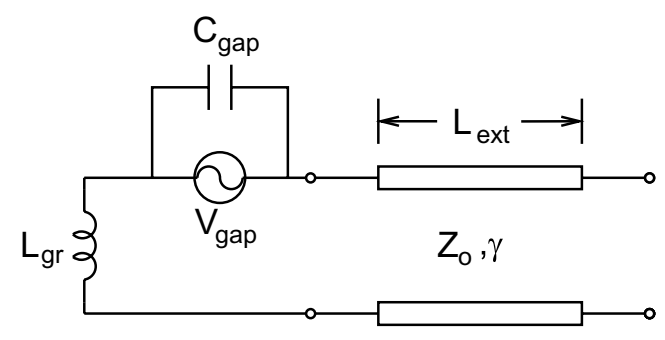

Fig. 4. Equivalent circuit model for the parasitic effects in the ground ring excitation scheme including an extension line of length $L_{e x t}$.

the ground conductors to the backside metallization. In the actual on-wafer situation the probe ground is connected to the backside metallization through some unknown environmental impedance $^{1}$. Furthermore the wave-port fingers extend the width of the ground planes thus altering the characteristics of the FG-CPW at the excitation reference plane. As a result the wave-port excitation will not resemble the GSG probe excitation applied during on-wafer measurements leading to the observed deviation. The surrounding ground ring simulation results shows good agreement with measurement in the lower microwave region. At higher frequencies the deviation is caused by parasitic effects and higher order modes associated with the excitation scheme. The simulation results from the metallic box wall setup shows a large deviation from measurements even at low frequency. In addition to the difficulties already described in connection with the surrounding ground ring, the metallic box wall setup suffers from a shift of the reference plane compared to the on-wafer measurements.

\section{Calibration Method for Accuracy ENHANCEMENT}

For the surrounding ground ring excitation scheme as shown in Fig. 2 a parasitic capacitance can be associated with the fringing field in the vicinity of the lumped port and a parasitic inductance with current flow along the PEC bridge. The equivalent circuit model for the excitation discontinuity shown in Fig. 4 is proposed, where the gap voltage source $V_{\text {gap }}$ is assumed to be shunted by a pure capacitance $C_{g a p}$, while the PEC bridge inductance $L_{g r}$ is assumed to be in series with the ground conductors of the FG-CPW extension line structure. This extension line is added at each port to allow the higher order modes generated by the lumped port to decay before reaching the reference plane for model extraction. The influence from the extended lines must subsequently be removed by de-embedding.

\section{A. Extended L-2L Calibration Method}

In the original L-2L calibration method [4] the port discontinuity is represented as a pure shunt capacitance. In this work an extended L-2L calibration method is introduced allowing to evaluate and remove also the series inductance, shown in Fig. 4. Two uniform lines of length $\mathrm{L}$ and $2 \mathrm{~L}$, respectively, are simulated using the ground ring excitation scheme in Ansoft

\footnotetext{
${ }^{1}$ This applies to the case without on-chip via-holes.
}
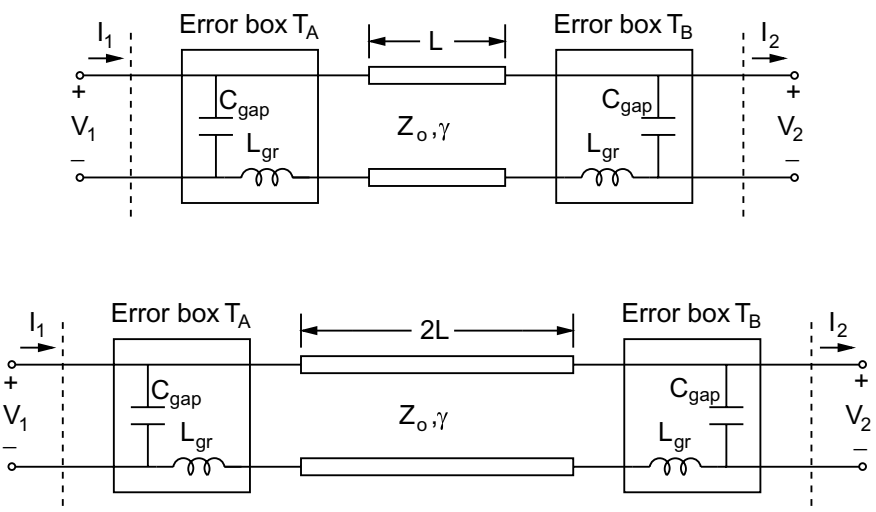

Fig. 5. The uniform $\mathrm{L}$ and $2 \mathrm{~L}$ calibration lines embedded with error boxes due to parasitic effects from the excitation scheme.

HFSS. The equivalent circuit model of the two calibration standards are shown in Fig. 5. Two error boxes $T_{A}$ and $T_{B}$ represent the parasitic effects associated with the excitation scheme.

Considering $A B C D$-parameters the product of the two error-boxes can be determined from

$$
T_{A} T_{B}=T_{t h r u}=T_{L, E M} T_{2 L, E M}^{-1} T_{L, E M}
$$

where $T_{L, E M}$ and $T_{2 L, E M}$ represent the ABCD-parameters obtained from simulation of uniform lines of lengths $\mathrm{L}$ and 2L, respectively. Assuming anti-symmetrical error boxes the parasitic elements in the equivalent circuit model shown in Fig. 4 can be extracted as

$$
C_{\text {gap }}=\frac{1}{2 \pi f} \Im\left\{\frac{C_{t h r u}}{1+A_{t h r u}}\right\} ; L_{g r}=\frac{1}{2 \pi f} \Im\left\{\frac{B_{t h r u}}{2}\right\}
$$

where $f$ is the frequency. The values of the gap capacitance $C_{\text {gap }}$ and ground inductance $L_{g r}$ associated with the surrounding ground ring excitation scheme are shown in Fig. 6. A nearly frequency independent gap capacitance $C_{g a p}$ of $6.9 \mathrm{fF}$ and ground inductance $L_{g r}$ of $27.7 \mathrm{pH}$ are observed for the parasitic elements validating the equivalent circuit model in Fig. 4. For accurate model extraction of small-size on-wafer passive components such parasitics can not be neglected.

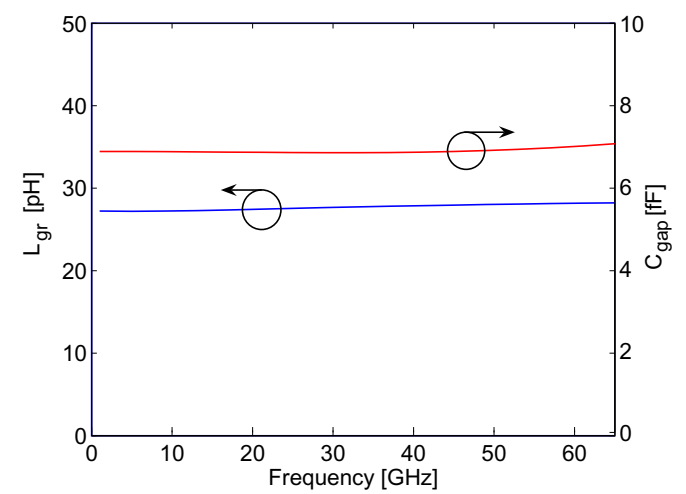

Fig. 6. Extracted ground ring parasitic values from extended L-2L calibration. 


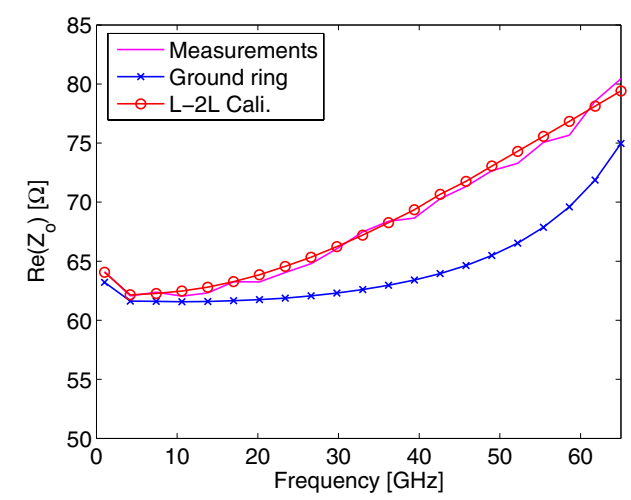

Fig. 7. Measured and simulated characteristic impedance for on-wafer interconnect line $(1 \mathrm{GHz}-65 \mathrm{GHz})$. Measurements (-), ground ring setup $(-\mathrm{x}-)$, ground ring setup with extended L-2L calibration (-o-).

Once the parasitics are known it is straightforward to remove their influence from the simulation result. To deembed the extension lines the characteristic impedance $Z_{o}$ and propagation constant $\gamma$ needed are determined from the corrected calibration line of length $\mathrm{L}$ or $2 \mathrm{~L}$.

\section{B. Accuracy Enhancement}

The characteristic impedance for the interconnect line extracted from the EM simulation result after the extended L-2L method has been applied is shown in Fig. 7. For comparison the characteristic impedance extracted from the uncorrected EM simulation result using the ground ring setup discussed in section III is repeated. The correlation with measurements has clearly improved especially at increased frequencies where the uncorrected approach are unable to predict the measurement results. It should be noticed that a removal of the parasitic gap capacitance $C_{g a p}$ and ground ring inductance $L_{g r}$ from the EM simulation results are not sufficient to obtain such accurate results. It is equally important to add the extension lines at each port to allow the higher order modes to decay to negligible levels before reaching the reference plane for model extraction.

In Fig. 8 the magnitudes of the measured S-parameters for the lumped $L C$-resonator are compared with the EM simulation results obtained with the surrounding ground ring excitation scheme before and after applying the extended L-2L calibration method. While both EM simulation results behave similarly up to approximately $25 \mathrm{GHz}$, the observed features in the magnitude of measured $S_{21}$ around the resonance frequency of $28 \mathrm{GHz}$ and measured $S_{11}$ around the frequency $55 \mathrm{GHz}$, respectively, can only be predicted by EM simulation using the accurate calibration method proposed here. A similar improvement in the prediction of the phases for the measured $\mathrm{S}$-parameters is also obtained.

\section{CONCLUSIONS}

We have demonstrated an efficient calibration procedure, which improve the accuracy of results from EM simulations. This procedure is especially useful for modeling of passive

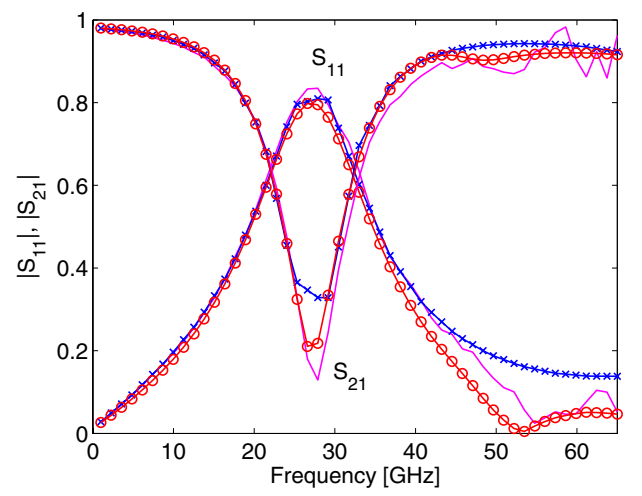

Fig. 8. Measured and simulated S-parameters for lumped $L C$-resonator (1 $\mathrm{GHz}-65 \mathrm{GHz}$ ). Measurements (-), ground ring setup (-x-), ground ring setup with extended L-2L calibration (-o-).

integrated components. It has been shown that in standard EM simulations the excitation ports give rise to discontinuities in the simulation domain, which can be modeled with an equivalent circuit comprised of a shunt capacitance and series inductance.

Comparison between measured and simulated results for an interconnect line and a lumped LC resonator provides excellent agreement if the simulation data is calibrated using the proposed model. Especially at increased frequencies this procedure ensures accurate results suitable for modeling purposes.

\section{ACKNOWLEDGMENT}

The authors would like to thank Kristoffer Sander from Ansoft Nordic for many fruitful discussions. This work was partly supported by the FPG-IST-project, GIBON.

\section{REFERENCES}

[1] F. Ling et al., "Systematic Analysis of Inductors on Silicon Using EM Simulations", Electronic Components and Technology Conference, pp. 484-488, 2002.

[2] D. G. Swanson Jr. and W. J. R. Hoefer, Microwave Circuit Modeling Using Electromagnetic Field Simulation, Artech House Inc., 2003.

[3] Ansoft Corporation, user's guide-High Frequency Structure Simulator, 2. edition, Mar. 2004.

[4] J. C. Rautio, "A De-Embedding Algorithm for Electromagnetics", Int. Jour. Microwave and Millimeter-Wave Computer-Aided Engineering, vol. 1, no. 3, pp. 282-287, 1991.

[5] L. Zhu, and K. Wu, "Unified Equivalent-Circuit Model of Planar Discontinuities Suitable for Field Theory-Based CAD and Optimization of M(H)MIC's", IEEE Trans. Microwave Theory \& Techn., vol. 47, no. 9, pp. 1589-1601 , Sept. 1999.

[6] L. Zhu, and K. Wu, "Network Equivalence of Port Discontinuity Related to Source Plane in a Deterministic 3-D Method of Moments", IEEE Microwave and Guided Wave Letters, vol. 8, no. 3, pp. 130-132, Mar. 1998.

[7] C-C. Tien et al., "Transmission Characteristics of Finite-Width Conductor-Backed Coplanar Waveguide", IEEE Trans. Microwave Theory \& Techn., vol. 41, no. 9, pp. 1616-1623, Sept. 1993.

[8] J. C. Rautio, "A New Definition of Characteristic Impedance", IEEE MTTS Digest, pp. 761-764, 1991. 